\title{
Informationsaustausch in religiösen Instituten
}

\author{
von Mark Reuver
}

Die grundsätzliche Bedeutung der Kommunikation für den Menschen als ein soziales Wesen wird heute nicht bestritten. Zwischenmenschliche Kommunikation und ihre Grundformen sind so alt wie die Menschheit selbst. Erst das Anwachsen der technischen Möglichkeiten zusammen mit einer verhältnismäßig starken Steigerung der Allgemeinbildung machen aber die zwischenmenschliche Kommunikation $\mathrm{zu}$ einer Macht, die als „öffentliche Meinung“ alle Lebensbereiche des Menschen erfaßt. Fast überall ist der Mensch heute dem ständigen Einfluß verschiedener Kommunikationssysteme ausgesetzt, und das läßt natüllich auch die Tür für Mißbräuche offen. Das Problem aber scheint heute nicht so sehr die strenge Kontrolle der sogenannten Massenmedien zu sein, sondern eher die Frage der schöpferischen und konstruktiven Entwicklung dessen, was mitgeteilt werden soll.

Als aktueller Vollzug des Lebens und der Lehre Christi hat die Kirche eine Botschaft für die Menschen aller Zeiten. Kommunikation ist ihr deswegen wesentlich. Grundsätzlich damit beauftragt, der Welt Leben, Wahrheit und Liebe mitzuteilen, sollte die Kirche eigentlich die Erste sein, die vom technischen Fortschritt der sozialen Kommunikationsmittel profitiert. Gerade sie sollte dadurch besser und wirksamer mit den Menschen in Verbindung und ins Gespräch kommen.

Im Dekret des Zweiten Vaticanums über die publizistischen Mittel hat sich zum ersten $\mathrm{Mal}$ in der Geschichte der Kirche überhaupt ein allgemeines Konzil zu Fragen der zwischenmenschlichen Kommunikation geäußert. Doch auch vor und nach dem Konzil haben die Päpste spätestens von Pius XI. bis zu Paul VI. ihr Interesse für die moderne Publizistik gezeigt. Zusammengenommen sind die Konzilsdokumente, besonders jene über die Religionsfreiheit, die Kirche in der Welt, über Dialog und Okumene auch für den Gebrauch und den Inhalt publizistischer Mittel von solcher Bedeutung, daß man der zwischenmenschlichen Kommunikation eine nicht geringe Rolle beimessen muß, wenn es darum geht, den neuen Pfingstgeist zu allen Menschen $\mathrm{zu}$ bringen.

Das Konzilsdekret über die publizistischen Mittel behandelt diese ausschließlich im Zusammenhang mit dem Apostolat. Es beschäftigt sich mit dem Dialog zwischen Kirche und Welt, mit der Sendung der Kirche zu allen Menschen und mit der pastoralen Bedeutung der publizistischen Mittel. Doch gibt es noch ein anderes Feld der Kommunikation, das all diesen Dingen vorgelagert und noch bedeutsamer ist: die Kommunikation „ad intra“, die allein Grundlage und Korrektiv für eine Kommunikation „ad extra“ ist.

\section{Offener Dialog in der Kirche}

Während das Dekret über die publizistischen Mittel sich nicht mit der Kommunikation innerhalb der Kirche beschäftigt, sieht die Pastoralkonstitution über die Kirche in der Welt eine freie und offene zwischenmenschliche Kommunikation unter den

P. Mark Reuver O. Carm., Lect. S. Th., Lic. Hist. Eccl., Rom, ist als Direktor des "Centrum Internationale Ordinis Carmelitani“ (Citoc) verantwortlich für die internen und externen publizistischen Aufgaben des Ordens. 
Gläubigen, den Dialog als Grundbedingung für die Sendung der Kirche in die Welt, an. Erst wenn innerhalb der Kirche der Dialog stattfindet, kann ein aufrichtiger Dialog der Kirchen mit der Welt und den Menschen aller Nationen, Rassen und Kulturen stattfinden: „Das aber verlangt von uns, daß wir vor allem in der Kirche selbst, bei Anerkennung aller rechtmäßigen Verschiedenheit, gegenseitige Hochachtung, Ehrfurcht und Eintracht pflegen, um ein immer fruchtbares Gespräch zwischen allen in Gang zu bringen, die das eine Volk Gottes bilden, Geistliche und Laien. Stärker ist, was die Gläubigen eint, als was sie trennt. Es gelte im Notwendigen Einheit, im Zweifel Freiheit, in allem die Liebe. “1

Ahnlich äußert sich Papst Paul VI. in seiner Enzyklika „Ecclesiam Suam“, der Magna Charta des Dialogs, in der er Natur und Ziel solcher Kommunikation innerhalb der Kirche folgendermaßen ausdrückt:

„Wie sehr wünschen wir, daß dieser häusliche Dialog in der Fülle des Glaubens und tätiger Liebe vor sich gehe, daß er mit Eifer und Familiengeist gepflegt werde, empfänglich für jede Wahrheit, jede Tugend, für alle uns überkommenen Schätze der Lehre und des geistlichen Lebens, daß er zutiefst durchdrungen sei von echter Frömmigkeit, bereit, die vielfältigen Anregungen unserer Zeit aufzugreifen, fähig, die Katholiken zu wahrhaft guten, weisen, freien, frohen und starken Menschen zu machen."2

Die Kommunikation innerhalb der Kirche hat in Laufe der Jahrhunderte nie ganz gefehlt. Schon die Apostelgeschichte konfrontiert uns mit einer Gemeinschaft, die diskutiert, sich beschwert, kritisiert und Vorschläge macht. Das Konzil von Jerusalem ist auch ein Ergebnis der „öffentlichen Meinung“ innerhalb der ersten christlichen Gemeinschaft, in der es verschiedene Ansichten über die Verpflichtungen der Heiden gab. ${ }^{3}$ Es gibt außerdem noch andere Anzeichen, aus denen klar wird, wie gerade die frühe Kirche den Dialog nicht nur erlaubte, sondern auch verstand, davon zu profitieren. Beim Weiheritus für Priester und Bischöfe werden noch heute die Umstehenden über ihre Meinung zur Würdigkeit der Weihekandidaten gefragt. Auch wenn dieser Ritus heute zu einer Formalität abgesunken ist, hatte er für die Gläubigen früherer Jahrhunderte doch eine reale Bedeutung. Für lange Zeit stand die Kirche unter dem Einfluß einer Gesellschaft, die formal nur Einbahnstraßen-Publizistik erlaubte. Nur eine Kommunikation zwischen jenen, die Macht hatten, war durch das Ancien Régime erlaubt. Kardinäle und Bischöfe, Könige und Prinzen, Botschafter und Mitglieder der High Society hatten allein das Privileg, über Ereignisse und Entwicklungen informiert zu werden. Nur ihre Meinung hatte Aussicht gehört zu werden. Im übrigen erhielt alles den Stempel des Geheimnisses, und selbst unbedeutende kirchliche Dekrete wurden als Wille Gottes dargestellt. Die Gläubigen durften keine Meinung haben, und die geringste Kritik wurde als Angriff auf das Wesen der Kirche ausgelegt.

Der übernatürliche Charakter der Kirche machte sie als Gesellschaft einmalig. Wohl darauf ist es zurückzuführen, daß sie, mehr oder weniger bewußt, der revolutionären Entwicklung der Technik und dem Einfluß der publizistischen Mittel im Grunde fernblieb. Die wachsende Informationslawine und die Bildung einer „öffentlichen Meinung", die um die Jahrhundertwende einen entscheidenden Punkt erreichten, konnten die Kirche nicht aufwecken. Sie ließ sich nicht warnen, als etwa Ivy Lee, einer der bedeutenden Mitbegründer der Public Relations, schon 1906 schrieb: „Unser Plan ist, kurz und offen, für Wirtschaftskonzerne und öffentliche Institutionen die Presse und die Bevölkerung schnell und genau über Tatsachen zu informieren, die für sie von Wert und Bedeutung sind. ${ }^{* 4}$ 
Viele Jahre blieb die Kirche als öffentliche Institution am Rande dieser Entwicklung. Papst Pius XII. brach 1950 den Bann mit Worten, die auch heute noch größte Aktualität besitzen. In seiner Botschaft an den dritten Internationalen Kongreß der katholischen Presse stellte der Papst fest, daß die „öffentliche Meinung“ zu jeder normalen Gesellschaft von Menschen, die sich ihrer persönlichen und sozialen Verantwortung bewußt sei, gehöre. Wo eine solche „öffentliche Meinung “ fehle, sagte der Papst, sei die Gesellschaft krank, ja verderbt. Dann kam er unmittelbar auf die Rolle der öffentlichen Meinung in der Kirche zu sprechen. Auch die Kirche sei, so sagte er, „ein lebendiger Organismus und in ihrem Leben würde etwas fehlen, wenn es in ihm nicht eine öffentliche Meinung gäbe, deren Fehlen den Seelsorgern und Gläubigen zur Last zu legen wäre".5 Der Sinn der Papstworte ist klar: Es gehört zur menschlichen Gesellschaft, ein Kommunikationssystem zu haben; die Mitglieder dieser Gesellschaft haben ein Recht auf Information, auf Kommunikation und darauf, eine „öffentliche Meinung " zu bilden.

Auch Papst Johannes XXIII. bestätigt in seiner Enzyklika „Pacem in Terris“ das natürliche Recht des Menschen, objektiv informiert $\mathrm{zu}$ werden und seine eigene Meinung $\mathrm{zu}$ sagen und $\mathrm{zu}$ verbreiten. ${ }^{6}$ Im Dekret über die publizistischen Mittel heißt es dazu: „Es gibt also in der menschlichen Gesellschaft ein Recht auf Information über alle Tatsachen, die den Menschen als einzelnen oder als Mitgliedern der Gesellschaft, je nach ihrer besonderen Situation, zu wissen zukommt. " 7 Im gleichen Dekret heißt es an anderer Stelle: „Die öffentliche Gewalt hat hier mit Rücksicht auf das Gemeinwohl, dem die sozialen Kommunikationsmittel zugeordnet sind, besondere Verpflichtungen. Im Rahmen ihrer Zuständigkeit hat sie die wahre und rechte Freiheit der Information, deren die heutige Gesellschaft zu ihrem Fortschritt bedarf, $\mathrm{zu}$ verteidigen und $\mathrm{zu}$ schützen, das gilt besonders für die Pressefreiheit."8

\section{Die Kirche - das Volk Gottes}

Wenn das Recht auf Information und „öffentliche Meinung“ Teil jeder menschlichen Gesellschaft ist und wenn der Fortschritt der modernen Gesellschaft weithin von der freien zwischenmenschlichen Kommunikation abhängt, dann gilt das in besonderem Maße von der Kirche. Aus soziologischer Sicht ist die Kirche eine vollkommene, öffentliche Gesellschaft mit repräsentativen Organen, die Autorität besitzen. Doch die Kirche ist noch mehr als eine Gesellschaft oder eine Institution. Christus hat sie in der Form einer Gemeinde eingesetzt, damit sie ein eigenes Volk bilde. Diese Gesellschaft muß der lebendigen Gemeinde jener dienen, die an Gottes Wort glauben und die selbst den mystischen Leib bilden.

Es ist nicht übertrieben, die ständige Betonung der grundsätzlichen Gleichheit aller Glieder der Kirche als das bedeutendste Charakteristikum des Zweiten Vatikanischen Konzils zu bezeichnen: ihre gemeinsame Sendung und die Verpflichtung aller, am vollen Leben und der vollen Aufgabe der Kirche teilzunehmen. Die freie Kommunikation und umfassende Information in allen irgendwie für die Mitglieder wichtigen Dingen liegt in der Natur einer solchen Kirchengemeinschaft. Sie ist von vitaler Notwendigkeit. Alle Glieder der kirchlichen Gemeinschaft empfangen Gottes Wort und die Sakramente, sie alle leben im Glauben und in der Gnade und führen so das Leben Christi in dieser Zeit, teilnehmend an seiner königlichen, priesterlichen und prophetischen Aufgabe, fort.

Alle Kirchenglieder zusammengenommen konstituieren diese Gemeinschaft, und zusammen sind sie verantwortlich für die Ankunft der Frohen Botschaft in jener 
Welt, in der sie selbst leben: „Ist doch ihre eigene Gemeinschaft aus Menschen gebildet, die, in Christus geeint, vom Heiligen Geist auf ihrer Pilgerschaft zum Reich des Vaters geleitet werden und eine Heilsbotschaft empfangen haben, die allen auszurichten ist. Darum erfährt diese Gemeinschaft sich mit der Menschheit und ihrer Geschichte wirklich engstens verbunden. "9

Mit der gleichen Erfahrung der Erdhaftigkeit hineingestellt in diese Welt ${ }^{10}$ hat die Kirche das Werk Christi voranzutragen: „In der Erfüllung dieses ihres Auftrages obliegt der Kirche allzeit die Pflicht, nach den Zeichen der Zeit zu forschen und sie im Lichte des Evangeliums zu deuten. " 11 Das Wort Gottes gibt wirklich eine Antwort auf alle Probleme der Zeit, doch ist diese Antwort bedingt durch die Geschichte, die Art der Probleme und ihre Umstände. „Es gilt also, die Welt, in der wir leben, ihre Erwartungen, Bestrebungen und ihren oft dramatischen Charakter $\mathrm{zu}$ erfassen und $\mathrm{zu}$ verstehen ".12

Die Antwort an die Welt von heute erfordert einen intensiven Kommunikationsaustausch zwischen allen Gliedern der Kirche, wenn man die Zeichen der Zeit verstehen und richtig deuten will. „Zur Steigerung dieses Austausches bedarf die Kirche vor allem in unserer Zeit mit ihrem schnellen Wandel der Verhältnisse und der Vielfalt ihrer Denkweisen der besonderen Hilfe der in der Welt Stehenden, die eine wirkliche Kenntnis der verschiedenen Institutionen und Fachgebiete haben und die Mentalität, die in diesem am Werke ist, wirklich verstehen, gleichgültig, $o b$ es sich um Gläubige oder Ungläubige handelt. Es ist jedoch Aufgabe des ganzen Gottesvolkes, vor allem auch der Seelsorger und Theologen, unter dem Beistande des Heiligen Geistes auf die verschiedenen Sprachen unserer Zeit zu hören, sie zu unterscheiden, zu deuten und im Lichte des Gotteswortes zu beurteilen, damit die geoffenbarte Wahrheit noch tiefer erfaßt, besser verstanden und passender verkündet werden kann. “13

Die Menschwerdung Christi vollendet sich in jedem Augenblick der Weltgeschichte. In dieser grundsätzlichen christlichen Perspektive gesehen, hat jedes Stück Information, jede Nachricht ihre Bedeutung und kann dazu beitragen, Gott, der die Welt erschaffen, und Christus, der sie erlöst hat, besser zu erfassen. ${ }^{14}$ Alle Glieder der lebendigen Kirchengemeinschaft haben die Pflicht, jene Ereignisse zu sehen, $\mathrm{zu}$ deuten und $\mathrm{zu}$ beurteilen, in denen sich die Geschichte der Erlösung bis zur Vollendung der Zeiten in Christus kundtut.

Die kirchliche Gemeinschaft selbst hat durch eine von Christus verliehene Autorität ein authentisches Urteil und eine echte Antwort in allen Phasen der Geschichte zu sichern. Dies alles geschieht jedoch innerhalb der kirchlichen Gemeinschaft, im Dienst für alle Mitglieder und alle Menschen. Im Lichte dieser Aufgabe der Kirche können und sollten auch Spannungen zwischen Macht und Gemeinschaft, zwischen Autorität und Freiheit, zwischen Kommunikation und „öffentlicher Meinung " gelöst werden. ${ }^{15}$ Nur ein offener und ehrlicher Dialog zwischen Pfarrern, Theologen, Spezialisten und den Christen allgemein kann alle Schwierigkeiten überwinden.

\section{Religiöse Institute in der Kirche}

Es ist nicht schwer, alles, was bisher gesagt wurde, auf die religiösen Institute, also auf Orden und Genossenschaften, anzuwenden. Jedes dieser Institute bildet mit seinen eigenen repräsentativen und autoritativen Organen eine Gesellschaft, und alle Mitglieder bilden zusammen eine echte Gemeinschaft. Die religiösen Institute 
sind, soweit sich ihre Glieder dem Leben nach dem Evangelium verschrieben und sich selbst ganz dem Dienste Christi geweiht haben, lebendiger Teil der Kirche. Sie identifizieren sich selbst mit der Sendung der Kirche, indem sie sich der Vollendung der Erlösung Christi für die Menschen und die Welt widmen.

Tatsïchlich hat aber die Geschichte manche dieser Elemente verdeckt. Manchmal haben solche Entwicklungen die religiösen Institute sogar daran gehindert, lebendige Kräfte innerhalb der Kirche zu sein. Man sieht sie heute kaum noch als treue, sichtbare und wirksame Zeichen Christi an, in denen sich sein Leben und seine Mission innerhalb der Kirche vollendet.

Das Charakteristikum des Religiosen, abgeschieden von der Welt zu sein, macht es für manche Institute schwer, offen zu sein für die Welt und mit der Kirche an der Bestimmung der Menschheit teilzunehmen. Jede bisherige starke Betonung der speziellen Aufgabe jedes Institutes förderte Partikularismus und Isolation. Sie half den einzelnen Mitgliedern kaum, sich zunächst als den Unternehmungen und Aufgaben der universalen Kirche verpflichtete Christen zu sehen. ${ }^{16}$

Die moralisch aszetische Deutung des Gelübdes des Gehorsams hat in der Vergangenheit einen Abgrund zwischen Oberen und Untergebenen, zwischen Autorität und Kommunität aufgerissen. Während eine religiöse Gerneinschaft eigentlich wie eine Familie sein sollte, die im Namen des Herrn beisammen sich seiner Gegenwart freut ${ }^{17}$, haben sich diese Institute tatsächlich $\mathrm{zu}$ "geschlossenen " Gesellschaften entwickelt, wo Geheimniskrämerei und nicht selten Intrigen die Mitglieder ernstlich daran gehindert haben, als Menschen ihre Persönlichkeit und auch ihre persönliche Verantwortung für die Sache der Kirche und der Welt zu entfalten.

Wenn inzwischen die Offnung nach innen und außen für die religiösen Institute alte Barrieren niedergerissen hat, dann bildet auch dazu das Zweite Vaticanum den offiziellen Wendepunkt. Die Erneuerung und Anpassung religiöser Institute ist weder eine Frage der Veränderung des Ordenskleides, noch einer schwungvollen Aufnahme des Zeitgeistes. Die Erneuerung besteht vielmehr in einer Änderung der Haltung und der Atmosphäre, die einer Wiederherstellung wahrhaft evangelischer Gemeinschaften mit einer großen Offenheit für die Mitglieder und für alles, was in der Welt existiert, dient. Das Leben in Gemeinschaft solle - so sagt das Konzil - beharrlich nach dem Beispiel der Urkirche, in der die Menge der Gläubigen ein Herz und eine Seele war, gepflegt werden. ${ }^{18}$

In religiösen Instituten ist die grundsätzliche Gleichheit aller Glieder ein hervorstechendes Charakteristikum: alle Glieder des Institutes sind verantwortlich für das Leben und die Aufgabe ihrer Gemeinschaft. Deswegen fordert das Konzil von den Kapiteln und Räten, daß sie „je auf ihre Weise die sorgende Teilnahme aller Mitglieder am Wohl des ganzen Institutes zum Ausdruck bringen ".19 Deswegen heißt es: "Die Oberen jedoch sollen in dem, was die Belange des ganzen Institutes betrifft, ihre Untergebenen in geeigneter Weise befragen und hören. "20 Denn die von der Kirche gew ünschte Erneuerung kann nicht erfolgreich sein und nicht erreicht werden ohne "die Zusammenarbeit aller Mitglieder eines Institutes"..21 Religiöse Institute brauchen Autoritäten und Obere wie jede andere menschliche Gesellschaft. Doch ist bemerkenswert, daß das Konzilsdekret über das Ordensleben für die Mitglieder der männlichen und weiblichen Institute nicht das Wort "Untergebene" gebraucht. Stattdessen wird von Mitgliedern (sodales) gesprochen. Auch im Lichte der anderen Konzilsdokumente zu Fragen der Autorität wird deutlich, daß die Oberen nicht die Aufgabe haben zu kommandieren, sondern daß sie gewählt wurden, um den Gliedern der Gemeinschaft zu dienen. 


\section{Die Notwendigkeit der Kommunikation}

Als Glieder einer menschlichen Gesellschaft, als Christen und als Mitglieder einer Gemeinschaft innerhalb der Kirche haben die Religiosen das Recht, gut und sachlich über alles informiert $\mathrm{zu}$ werden, was für das Leben ihrer Institute irgendwie von Bedeutung sein kann. Ebenso haben sie das Recht, ihre Meinung zu äußern, mit ihren Mitbrüdern bzw. Mitschwestern und auch mit den Oberen den Dialog zu führen. Gerade ihre Teilnahme an der pastoralen Aufgabe der Kirche erfordert eine entsprechende Kommunikation unter den einzelnen Mitgliedern. Mit der Kirche selbst haben die religiösen Institute auf die Ängste, Notwendigkeiten und Wünsche der heutigen Welt zu antworten. Die Mitglieder jeder religiösen Gemeinschaft haben in gemeinsamer Arbeit die Zeichen der Zeit zu befragen, um in der ihnen besonderen Weise evangelischen Lebens eine Antwort auf bestimmte Probleme des Menschen unserer Zeit zu geben. Die ganze religiöse Gemeinschaft ist verantwortlich für diese Aufgabe. Deswegen müssen alle nach entsprechenden Wegen und Methoden suchen. Die Gemeinschaften sollten zusammen die notwendigen Planungen anstellen, um den Notwendigkeiten der heutigen Welt zu entsprechen. Freie und objektive Information, freier Gedankenaustausch zwischen Oberen und Mitgliedern und zwischen den Gliedern untereinander sind deswegen von wesentlicher Bedeutung für die religiösen Institute, wenn man jenen Standard erreichen will, den Kirche und Welt heute von ihnen verlangen. Kommunikation und Bildung einer „öffentlichen Meinung “ innerhalb der religiösen Gemeinschaften sind nicht eine Frage der Erlaubtheit, sondern eine durch die Natur dieser Gemeinschaften geforderte notwendige Grundlage, damit sie am Leben der Kirche teilnehmen und das Werk Christi in der Welt verbreiten können.

\section{Vielbeit und Einbeit}

Mit einer Bewegung zu größerer Dezentralisation und Pluralität verbindet sich heute ein starkes Bedürfnis nach Einheit. Die wachsende Erkenntnis von einer bestimmten, begrenzten Kultur, die aus bestimmten sozialen und ethnologischen Gegebenheiten erwächst, geht Hand in Hand mit dem Bewußtsein, zur einen Menschheit zu gehören, für deren Existenz alle Menschen verantwortlich sind. Die Dekrete des Zweiten Vaticanums sehen beide Bewegungen und nehmen sie an, um die Sendung der Kirche in die heutige Welt erfolgreich durchzuführen. Die Kirche vollendet Christi Leben und Werk gerade, indem sie sich selbst lebt und annimmt, indem sie sich selbst integriert in nationale, regionale und lokale Voraussetzungen und Notwendigkeiten. Diese beiden Bewegungen haben eine stark empfundene Rückwirkung auf die heutigen religiösen Institute. Es ist sicher ein gesundes Zeichen, wenn sie sich heute gleichzeitg dem Trend nach Zentralisation und Dezentralisation ausgesetzt sehen. Die eine zentrifugale Bewegung festigt und stärkt die Autonomie, die Subsidiarität und Pluriformität, die jene Aufgabe und Verantwortung des einzelnen, jeder Gemeinschaft innerhalb der verschiedenen Kulturen und Lebensweisen, innerhalb der verschiedenen Bedürfnisse und Wünsche lokaler Kirchen kennzeichnet. Die andere zentripetale Bewegung sucht das allgemeine Gut, die gemeinsame Aufgabe und die Aufgabe des gesamten Institutes innerhalb der Kirche und der Welt von heute. Die vielen Studienkongresse, Versammlungen und Kapitel unserer Tage sind ein sprechendes Zeugnis für diese beiden Trends nach Einheit und Vielheit.

Manche sprechen heute von einer bedrohlichen Krise der religiösen Institute. Sicherlich gibt es Schwierigkeiten und Probleme. Doch sind sie nicht notwendig das Ergeb- 
nis von weniger Respekt vor der Autorität, vor Oberen oder gar die Folge des Ungehorsams. Die religiösen Institute sind heute genau wie die Kirche selbst starken und tiefgreifenden Änderungen unterworfen, die einen neuen Abschnitt in der Weltgeschichte einleiten. ${ }^{22}$ Die neue Zeit erfordert einen neuen Stil christlichen und religiösen Lebens. ${ }^{23}$

In unseren Tagen einer solch durchgreifenden und schnellen Entwicklung brauchen wir vor allem die Kommunikation und die Entwicklung einer entsprechenden "öffentlichen Meinung" in der Kirche und in den religiösen Instituten. Wo Intoleranz und Druck die Atmosphäre vergiften, kann nur eine ehrliche Kommunikation innerhalb der Institute, zwischen Oberen und Gliedern, zwischen den Provinzen und den Mitgliedern untereinander den notwendigen Dialog bringen, der diese Gemeinschaften in die moderne Welt einfügt. Es ist selbstverständlich, daß eine solche zwischenmenschliche Kommunikation ihren Ursprung und ihre Lebensfähigkeit in einer Atmosphäre der Freiheit haben muß, die auch die Möglichkeit der Kritik einschließt. Solange die Religiosen unter dem Gesetz der Schwäche und Sünde stehen, kann Kritik eine erneuernde und reinigende $\mathrm{Kraft}$ sein. Dies ist allerdings nur dann der Fall, wenn Freiheit und Loyalität gegenüber den Oberen und gegenüber Sinn und Aufgabe des betreffenden Institutes zusammengehen.

Kommunikation ist ein Zwiegespräch und nicht eine Einbahnstraße ${ }^{24}$; sie ist ein Weg, der in beiden Richtungen begangen werden muß: vom Oberen und von den Mitgliedern der Genossenschaften und Orden, um eine entsprechende „öffentliche Meinung" über die anstehenden Fragen des Lebens und der Aufgabe dieser Gemeinschaften zu bilden.

\section{Beginn einer Verwirklichung}

Die Notwendigkeit einer entsprechenden Kommunikation innerhalb der religiösen Institute wird heute mehr und mehr empfunden. In verschiedenen Teilen der Welt wurden bereits entsprechende Kommunikationszentren eröffnet. Interne Ordensprovinz-Zeitschriften, Nachrichtenblätter und Nachrichtendienste haben ihre ersten zögernden Schritte innerhalb von Ordensgemeinschaften getan. Der Austausch von Ideen und Meinungen - durch persönliche Initiativen einzelner oder von Provinzialen begonnen - verstärkt sich. Auch in Rom, wo die meisten religiösen Institute ihre Generalate haben, sind die Dinge in Bewegung. ${ }^{25}$ Einige wenige Institute haben den neuartigen Gedanken und den Mut gehabt, internationale Zentralbüros für interne Kommunikation einzurichten. Alles, was hier bisher gesagt wurde, macht deutlich, daß jede religiöse Gemeinschaft ein solches Zentrum für interne Kommunikation braucht. Allerdings kann dies nur unter der Bedingung geschehen, daß ein solches Zentrum frei ist und nicht nur jene Dinge weitergibt, die "von oben" diktiert werden. Nur in Freiheit kann ein Kommunikationsbüro Oberen und Mitgliedern einer Gemeinschaft wirklich dienen. Bestimmt von der Loyalität gegenüber den Oberen kann es entscheidende Hilfe leisten, ein gutes und zutreffendes Image von der zentralen Verwaltung einer Gemeinschaft zu bilden. Andererseits sollte eine solche Stelle ihre Verpflichtung sehen, die zentrale Leitung über alles zu informieren, was aus dem Institut, der Kirche und der Welt für sie von Interesse ist, wo und wie ihre Mitglieder einer neuen Situation, neuen Problemen gegenüberstehen und wie das gleiche Leben innerhalb des gleichen Institutes sich in verschiedenen Formen ausdrücken kann. Die Orientierung des Institutes durch den Oberen sollte so gegeben werden, daß sie bereitwillig angenommen wird, und gleichzeitig sollten 
die Oberen darüber informiert werden, wie ihre Direktiven am günstigsten den Weg zu den einzelnen Gemeinschaften finden, indem sie deren örtlichen Gegebenheiten und Bedürfnissen entsprechen. Es ist Aufgabe eines solchen internationalen Konmunikationszentrums, die Kommunikation und den Dialog unter den Provinzen und den Mitgliedern zu pflegen. Es sollte helfen, neuen Initiativen und schöpferischen Erneuerungen zum Durchbruch zu verhelfen. Alle persönlichen und gemeinschaftlichen Initiativen können positive Hilfe sein und dazu beitragen, das Ziel der betreffenden Gemeinschaft besser zu erfüllen, wenn sie allen Mitgliedern des betreffenden Institutes bekannt werden. Ein Kommunikationssystem innerhalb religiöser Institute ist ein Instrument, das sowohl den Generalaten als auch allen Mitgliedern hilft, nach neuen Wegen religiösen Lebens zu suchen, wo alle Religiosen den Mut finden, offen zu sein für die Charismen des evangelischen Lebens.

\section{Anmerkungen:}

1. Zweites Vatikanisches Konzil, Pastorale Konstitution über die Kirche in der Welt „Gaudium et Spes“, 92

2. Paul VI., Enzyklika „Ecclesiam suam“ vom 6. 8. 1964, Acta Apostolicae Sedis, Vatikan, 56:1964, 657 f. Vgl. audh u. a.: Herder-Korrespondenz, Freiburg, 18:1963/64, 583

3. Emile Gabel, A. A. L'opinion publique dans l'Eglise, in: L'opinion publique, Semaine Sociales de France, Lyon 1966, 365

4. Zitiert bei: Herbert Lloyd, Public Relations, London 1963, 3; vgl. ferner: Albert Oeckl, Handbuch der Public Relations, München 1964, 89

5. Pius XII., Ansprache an den 3. Internationalen Kongreß der katholischen Presse vom 18. Februar 1950, Acta Apostolicae Sedis, Vatikan, 42:1950, 256 vgl. Radio Vaticana (Hrsg.), Documenti Pontifici sulla Stampa, Vatikan 1964, Nr. 65, 402

6. Johannes XXIII., Enzyklika "Pacem in terris" vom 11. 4. 1963, Acta Apostolicae Sedis, Vatikan, 55:1963, 257-304

7. Zweites Vatikanisches Konzil, Dekret über die publizistischen Mittel „Inter Mirifica“, 5

8. ebda. 12

9. Zweites Vatikanisches Konzil, Pastorale Konstitution über die Kirche in der Welt "Gaudium et Spes ${ }^{\alpha}, 1$

10. vgl. ebda, 40

11. ebda. 4

12. ebda.

13. ebda. 44

14. vgl. M. D. Chenu OP, „Vox Populi - Vox Dei“, Vortrag auf einem Symposion über christliche Information, veranstaltet von IDO-C und vom Weltrat der Kirchen, 6. - 8. 10. 1967 in Rom. Deutscher Text in: „Medium, Zeitschrift für evangelische Rundfunk- und Fernseharbeit "', München, 4:1967, 253-266.

15. Vgl. Marco Reuver, Publizistischer Arbeitskreis bein Weltlaienkongreß in: „Communicatio Socialis", Zeitschrift für Publizistik in Kirche und Welt, Emsdetten, 1:1968, 46 f. - Ferner: Caroline Cohen, Symposion Kirche und öffentliche Meinung, ebda. $48 \mathrm{f}$.

16. Zweites Vatikanisches Konzil, Dekret über die zeitgemäße Erneuerung des Ordenslebens "Perfectae Caritatis“, 2

17. ebda. 15

18. ebda. vgl. Apg. 4, 32

19. ebda. 14

20. ebda. 4. Bei diesem Satz handelt es sich um einen von 417 Konzilsvätern eingereichten Modus, der die Eröffnungsforderung dieses Abschnittes („Zur wirksamen Erneuerung und echten Anpassung ist die Zusammenarbeit aller Mitglieder eines Institutes unerläßlich") nicht zurücknehmen oder illusorisch machen soll. Vgl. F. Wulf, Dekret über 
die zcitgemäße Erneuerung des Ordenslebens, Kommentar in: Das Zweite Vatikanische Konzil, Lexikon für Theologie und Kirche, Band II, Freiburg 1967, 274 f. Man vergleiche dort auch die weiteren kritischen Anmerkungen zu diesem Text.

21. ebda. 4

22. Zweites Vatikanisches Konzil, Pastoralkonstitution über die Kirche in der Welt "Gaudium et Spes", 4

23. Sister Pearsons, Frances Loyola u. a. (Hrsg.), The New Nuns, Washington 1967, 141

24. Man vgl. hier auch die Entwicklung innerhalb der Publizistikwissenschaft, auf die u. a. O. B. Roegele in seinem Artikel "Kirche und Kommunikationsmittel in und nach dem Konzil“ (in: „Lebendige Kirche“, Mitteilungsblatt 6 des Diözesankomitees der Katholikenausschüsse der Erzdiözese Köln, Köln 1965/66, 6-14) verweist. Sie äußert sich etwa in den neuen Formulierungen vom „Zeitgespräch der Gesellschaft" (Arbeitsformel der Münchener Zeitungswissenschaft) oder im Begriff der „funktionalen Publizistik" (Prakke). Das Verhältnis zwischen Publizist und Publikum, so meint ctwa Henk Prakke, lasse sich ,am besten mit dem Bild eines Dialogs zwischen gleichberechtigten Partnern ausdrücken, die sich auf einer horizontalen Ebene im gesellschaftlichen Zwiegespräch begegnen " (Kommunikation der Gesellschaft, Münster 1967. 58; vgl. ders.: Alle Publizistik ist Zwiegespräch, in: „Publizistik“, Bremen, 5:1960, 208-210.)

25. Beispiel einer ähnlichen Entwicklung ist z. B. jener private Zusammenschluß von bisher 27 Generalsuperioren missionierender Orden in Rom, der unter der Bezeichnung SEDOS (Servizio di Documentatione e Studi) entstand und dem u. a. auch eine Arbeitsgemeinschaft für Publizistik zugeordnet ist. Zur Entstehung und Entwicklung von SEDOS vgl. Vincent Fecher svd, "Sedos spells Cooperation" in: The Word in the World 1968, Divine Word Missionaries, Techny/Ill. 1968, 174--176.

\section{S U M M A R Y}

The Council Decree on Social Communications silences dialogue and public opinion in the Church. But the Church's communication "ad intra" is previous to communication "ad extra", and conditions it. The natural right of members of a society to information and communication also concerns the Church as society. The Church, however is more than a society: the People of God. In the Church, all the members are fundamentally equal in their accepting God's word and the Sacraments, in their living the faith. All are committed to the Church's mission, and only through dialogue and communication the Church-community can continue the Incarnation and Redemption of Christ in this world. This calls for a new style of Christian and a new style of religious. The religious institutes share in the characteristic notes of Church-society and Church-community. Information, communication and public opinion are vital and most necessary for religious institutes. Religious institutes are drawn towards centralization and decentralization at the same time. In each institute a trend towards autonomy, subsidiarity and pluriformity goes hand in hand with the members' search for the common good, and the institute's common mission and scope in Church and world today. A two-way-communication will help to overcome intolerance and pressure which are characteristic of the present profound and rapid evolution. Communication systems with international centers in Rome, close to the Generalates, will be of great importance to aid the institutes to find the proper answers to problems in the world-Church. Freedom of information and communication together with loyalty towards the central government of the institute, openness with regard to new realities and initiatives, should contribute to discover the signs of the times and the answer which each particular institute has to give, inspired by the charism of the Gospel. (Full English text of this article is available through the Rome editor) 


\title{
RESUMEN
}

El Decreto del Concilio sobre las Comunicaciones Sociales no hable del diálogo y de las opiniones públicas en la Iglesia. Pero, la comunicación de la Iglesia nad intra" es anterior y condición de la comunicación „ad extra“.

El derecho natural de los miembros de una Sociedad a información y comunicación se refiere también a la Iglesia como sociedad. Sin embargo, la Iglesia es algo más que una sociedad: es el pueblo de Dios. En la Iglesia, todos los miembros que la componen, son fundamentalmente iguales al aceptar la palabra de Dios y los sacramentos, en la vida de la fe. Todos están obligados a la misión de la Iglesia, y sólo a través del diálogo y la comunicación la comunidad eclesial puede continuar la Encarnación y Redención de Christo en este mundo.

Esto exige un nuevo estilo de cristiano y un nuevo estilo de religiosos. Los Institutos religiosos participan en las notas características de la Iglesia-Sociedad e Iglesia-Comunidad. La información, comunicación y opinión pública son vitales y absolutamente necesarias para los Institutos religiosos. Los institutos religiosos se sienten atraidos hacia la centralización y descentralización al mismo tiempo. En todos los institutos, la tendencia hacia la autonomía, subsídiaridad y pluriformidad va acompañada de la búsqueda, por parte de los miembros, del bien común y de la misión común del instituto y finalidad de la Iglesia en el mundo actual. Una doble comunicación ayudará a vencer la intolerancia y la presión que son carasterísticas de la profunda y rápida evolución presente. Sistemas da comunicación con centros internacionales en Roma, cercanos a las sedes de los Generalatos, serán de gran importancia para ayudar a los Institutos a encontrar las respuestas adecuadas a los problemas del Mundo-Iglesia.

Libertad de información y comunicación, junto con lealtad hacia el gobierno central del Instituto, apertura en lo referente a las nuevas realidades e iniciativas, contribuirían a descubrir las señales de los tiempos y la respuesta que debe dar cada Instituto, inspirado por el carisma del Evangelio.

\section{Probleme der Hörfunkpredigt (II)}

\author{
von Ambrosius K. Ruf
}

\begin{abstract}
Von den „Spezifischen Schwierigkeiten des Hörfunkpredigers" bandelte ein erster Teil dieses Beitrags von Ambrosius $K$. Ruf in Nr. 2 von CS, der im folgenden mit den restlichen beiden Kapiteln abgeschlossen wird. Summary und Resumen beziehen Kapitel I mit ein.
\end{abstract}

\section{Regeln für den Hörfunkprediger}

Auf dem Hintergrund der vorausgegangenen Überlegungen soll jetzt von den besonderen Aufgaben des Hörfunkpredigers gesprochen werden; dabei ist nach Möglichkeiten zu suchen, wie die genannten Schwierigkeiten überwunden werden können. Es scheint, daß vier Regeln von jedem beachtet werden müssen, der über den Rundfunk eine große Hörergemeinde ansprechen will und der versucht, über dieses Medium die Frohbotschaft in den Raum der Welt zu tragen. 